\title{
On extension of fuzzy measures to aggregation functions
}

\author{
Anna Kolesárová ${ }^{1}$ Andrea Stupňanová ${ }^{2}$ Juliana Beganová $^{2}$ \\ ${ }^{1}$ Institute IAM, FCHFT, Slovak University of Technology, \\ Radlinského 9, 81237 Bratislava, Slovakia \\ e-mail: anna.kolesarova@stuba.sk \\ ${ }^{2}$ Faculty of Civil Engineering, Slovak University of Technology, \\ Radlinského 11, 81368 Bratislava, Slovakia \\ e-mail: \{andrea.stupnanova@stuba.sk;juliana.beganova@stuba.sk\}
}

\begin{abstract}
In the paper we study a method extending fuzzy measures on the set $N=\{1, \ldots, n\}$ to $n$-ary aggregation functions on the interval $[0,1]$. The method is based on a fixed suitable $n$-ary aggregation function and the Möbius transform of the considered fuzzy measure. This approach generalizes the wellknown Lovász and Owen extensions of fuzzy measures. We focus our attention on the special class of $n$-dimensional Archimedean quasi-copulas and prove characterization of all suitable $n$-dimensional Archimedean quasi-copulas. We also present a special universal extension method based on a suitable associative binary aggregation function. Several examples are included.
\end{abstract}

Keywords: Aggregation function, Choquet integral, copula, fuzzy measure, $n$-monotone function, quasi-copula, Archimedean quasi-copula

\section{Introduction}

In [13] we have introduced a method extending any fuzzy measure on the set $N=\{1, \ldots, n\}$ to an $n$-ary aggregation function by means of a (fixed) suitable aggregation function and the Möbius transform of the considered fuzzy measure. Recall that a fuzzy measure $m$ on the set $N=\{1, \ldots, n\}$ is a nondecreasing set function $m: 2^{N} \rightarrow[0,1]$ with the properties $m(\emptyset)=0$ and $m(N)=1$. An n-ary aggregation function $(n \in \mathbb{N}, n \geq 2)$ on the interval $[0,1]$ is a function $A:[0,1]^{n} \rightarrow[0,1]$ which is non-decreasing in each variable and satisfies the boundary conditions $A(\mathbf{0})=0$ and $A(\mathbf{1})=1$. We briefly outline the proposed method.

To any fuzzy measure $m$ on $N$ and a given fixed $n$ ary aggregation function $A$ we assign the function $F_{m, A}:[0,1]^{n} \rightarrow \mathbb{R}$ defined by

$$
F_{m, A}\left(x_{1}, \ldots, x_{n}\right)=\sum_{I \subseteq N} M_{m}(I) A\left(\mathbf{x}_{I}\right),
$$

where $M_{m}: 2^{N} \rightarrow \mathbb{R}, M_{m}(I)=$ $\sum_{K \subseteq I}(-1)^{|I \backslash K|} m(K), \quad$ is the Möbius transform of $m$ and $\mathbf{x}_{I}=\left(u_{1}, \ldots, u_{n}\right)$ is the $n$-tuple assigned to an input $n$-tuple $\mathbf{x}=\left(x_{1}, \ldots, x_{n}\right) \in[0,1]^{n}$ and a subset $I$ of the set $N$ by

$$
u_{i}= \begin{cases}x_{i} & \text { if } i \in I \\ 1 & \text { otherwise. }\end{cases}
$$

We say that the function $F_{m, A}$ extends the fuzzy measure $m$ if $\left.F_{m, A}\right|_{\{0,1\}^{n}}=m$. However, in general, the functions $F_{m, A}$ defined by (1) are neither aggregation functions (monotonicity can fail) nor extensions of $m$. Denote the set of all $n$-ary aggregation functions by $\mathcal{A}_{(n)}$ and the set of all fuzzy measures on $N$ by $\mathcal{M}_{(n)}$. In [13] we have completely characterized all aggregation functions which are suitable for this construction, i.e., all $A \in \mathcal{A}_{(n)}$ which together with any fuzzy measure $m \in \mathcal{M}_{(n)}$ give via (1) an aggregation function $F_{m, A}$ extending $m$. Such aggregation functions $A \in \mathcal{A}_{(n)}$ are briefly called suitable aggregation functions.

For example, all $n$-dimensional copulas are suitable aggregation functions. On the other hand, not all $n$-dimensional quasi-copulas possess this property. In this contribution we focus our attention on the special class of $n$-dimensional Archimedean quasicopulas and prove characterization of all suitable $n$-dimensional Archimedean quasi-copulas.

Our approach was motivated by the Lovász and Owen extensions of fuzzy measures, [14, 21]. If $A=$ $\operatorname{Min}$, where $\operatorname{Min}\left(x_{1}, \ldots, x_{n}\right)=\min \left\{x_{1}, \ldots, x_{n}\right\}$, then the expression on the right-hand side of formula (1) coincides with the the expression

$$
\sum_{I \subseteq N} M_{m}(I) \min _{i \in I} x_{i}
$$

which is equal to the Choquet integral $C-\int_{N} \mathbf{x} \mathrm{d} m$, [5], and also to the Lovász extension of the fuzzy measure $m$, [15]. Similarly, for $A=\Pi$, where $\Pi\left(x_{1}, \ldots, x_{n}\right)=x_{1} \cdots x_{n}$, the expression on the right-hand side of formula (1) coincides with the the expression

$$
\sum_{I \subseteq N} M_{m}(I) \prod_{i \in I} x_{i}
$$

which is known as the Owen extension of $m$. Note that the Lovász and Owen extensions can be applied universally, for any arity $n$, while the proposed 
method produces (if $A$ is a suitable $n$-ary aggregation function) $n$-ary aggregation functions extending $m$. In the last section a special universal extension method based on a suitable associative binary aggregation function is proposed. The method is illustrated by examples.

\section{Characterization of suitable aggregation functions}

As mentioned above, in [13] we have characterized all suitable aggregation functions $A$. For completeness of information let us recall two main results. The first of them characterizes all aggregation functions $A \in \mathcal{A}_{(n)}$ for which $F_{m, A}$ is an extension of $m$ for each $m \in \mathcal{M}_{(n)}$.

Theorem 1 Let $A \in \mathcal{A}_{(n)}$. The function $F_{m, A}$ defined by (1) is for each $m \in \mathcal{M}_{(n)}$ an extension of $m$ if and only if $A$ is an aggregation function with zero annihilator.

Recall that 0 is the annihilator of $A$ if $A\left(x_{1}, \ldots, x_{n}\right)=0$ whenever $0 \in\left\{x_{1}, \ldots, x_{n}\right\}$.

In general, extensions $F_{m, A}$ need not be monotone. Before giving conditions ensuring the monotonicity of $F_{m, A}$, we introduce some notations.

Fix $i \in N$. Let $\mathbf{u}, \mathbf{v}$ be any elements in $[0,1]^{n}$, such that

$$
\begin{aligned}
& \mathbf{u}=\left(x_{1}, \ldots, x_{i-1}, x_{i}, x_{i+1}, \ldots, x_{n}\right), \\
& \mathbf{v}=\left(x_{1}, \ldots, x_{i-1}, x_{i}^{\prime}, x_{i+1}, \ldots, x_{n}\right),
\end{aligned}
$$

with $x_{i}^{\prime}>x_{i}$. For any subset $E \subseteq N \backslash\{i\}$ denote by $\mathbf{u}^{E}$ and $\mathbf{v}^{E} n$-tuples with coordinates

$$
\begin{array}{ll}
u_{i}^{E}=x_{i} & v_{i}^{E}=x_{i}^{\prime}, \\
u_{j}^{E}=x_{j} & v_{j}^{E}=1, \quad \text { if } j \notin E \cup\{i\}, \\
u_{j}^{E}=0 & v_{j}^{E}=x_{j}, \quad \text { otherwise. }
\end{array}
$$

Finally, recall that for an $n$-ary aggregation function $A$, the $A$-volume of an $n$-box $[\mathbf{u}, \mathbf{v}]$ in $[0,1]^{n},[\mathbf{u}, \mathbf{v}]=$ $\left[u_{1}, v_{1}\right] \times \ldots \times\left[u_{n}, v_{n}\right]$, is defined by

$$
V_{A}([\mathbf{u}, \mathbf{v}])=\sum(-1)^{\alpha(\mathbf{c})} A(\mathbf{c}),
$$

where the sum is taken over all vertices $\mathbf{c}=$ $\left(c_{1}, \ldots, c_{n}\right)$ of the $n$-box $[\mathbf{u}, \mathbf{v}]$ (i.e., each $c_{k}$ is equal to either $u_{k}$ or $\left.v_{k}\right)$, and $\alpha(\mathbf{c})$ is the number of indices $k^{\prime} s$ such that $c_{k}=u_{k}$.

Theorem 2 Let $A \in \mathcal{A}_{(n)}$ be an aggregation function with zero annihilator. The function $F_{m, A}$ is for each $m \in \mathcal{M}_{(n)}$ non-decreasing in the ith variable $(i=1, \ldots, n)$ if and only if for all $n$ tuples $\mathbf{u}=\left(x_{1}, \ldots, x_{i-1}, x_{i}, x_{i+1}, \ldots, x_{n}\right), \mathbf{v}=$ $\left(x_{1}, \ldots, x_{i-1}, x_{i}^{\prime}, x_{i+1}, \ldots, x_{n}\right)$ in $[0,1]^{n}$ with $x_{i}^{\prime}>$ $x_{i}$, it holds that for each $E \subseteq N \backslash\{i\}, A$ volumes $V_{A}\left(\left[\mathbf{u}^{E}, \mathbf{v}^{E}\right]\right)$, where the end-points of $n$ boxes $\left[\mathbf{u}^{E}, \mathbf{v}^{E}\right]$ are defined by (2), are non-negative.
For example, by Theorem 2, for a binary aggregation function $A$ with zero annihilator the function $F_{m, A}$ is non-decreasing for each $m \in \mathcal{M}_{(2)}$ if and only if $A$-volumes of all possible 2-boxes $\left[\left(x_{1}, x_{2}\right),\left(x_{1}^{\prime}, 1\right)\right]$ and $\left[\left(x_{1}, x_{2}\right),\left(1, x_{2}^{\prime}\right)\right]$ in $[0,1]^{2}$ with $x_{1}^{\prime}>x_{1}$ and $x_{2}^{\prime}>x_{2}$, are non-negative. Note that $A$-volumes of 2-boxes of the type $\left[\left(0, x_{2}\right),\left(x_{1}, x_{2}^{\prime}\right)\right]$ and $\left[\left(x_{1}, 0\right),\left(x_{1}^{\prime}, x_{2}\right)\right]$, which in binary case are also obtained from conditions (2), are trivially nonnegative because of the monotonicity of $A$.

From Theorems 1 and 2 we obtain the following characterization.

Corollary 1 An n-ary aggregation function $A$ is a suitable aggregation function if and only if it has zero annihilator and satisfies the conditions for the monotonicity of $F_{m, A}$ given in Theorem 2 for each variable.

For example, all $n$-copulas [24, 19] are suitable aggregation functions for our construction. Recall that $n$-copulas are defined as functions $C:[0,1]^{n} \rightarrow$ $[0,1]$ satisfying

(C1) the boundary conditions:

if $0 \in\left\{x_{1}, \ldots, x_{n}\right\}$ then $C\left(x_{1}, \ldots, x_{n}\right)=0$, $C\left(1, \ldots, 1, x_{j}, 1, \ldots, 1\right)=x_{j}$ for each $j=$ $1, \ldots, n$ and each $x_{j} \in[0,1]$,

(C2) the $n$-increasing property:

$V_{C}([\mathbf{u}, \mathbf{v}]) \geq 0$ for each $n$-box $[\mathbf{u}, \mathbf{v}]$ in $[0,1]^{n}$.

It is easy to see that aggregation functions described in the following proposition also possess zero annihilator and the $A$-volumes of all $n$-boxes in $[0,1]^{n}$ are non-negative.

Proposition 1 Let $C$ be an $n$-copula, $f_{i}:[0,1] \rightarrow$ $[0,1]$, non-decreasing functions such that $f_{i}(0)=$ $0, f_{i}(1)=1, i=1, \ldots, n$. Then the function $A:[0,1]^{n} \rightarrow[0,1]$ defined by

$$
A\left(x_{1}, \ldots, x_{n}\right)=C\left(f_{1}\left(x_{1}\right), \ldots, f_{n}\left(x_{n}\right)\right),
$$

is a suitable $n$-ary aggregation function.

Not only copulas and distorted copulas from Proposition 1 are suitable aggregation functions.

Example 1 Consider the function $A:[0,1]^{3} \rightarrow$ $[0,1]$, given by

$$
A(x, y, z)=x y z \min (1, x+y+z) .
$$

The function $A$ is a ternary aggregation function with zero annihilator. It is not a copula, because, e.g., for $\mathbf{u}=(0.3,0.3,0.3)$ and $\mathbf{v}=$ $(0.35,0.35,0.35)$ the $A$-volume of the corresponding 3 -box is $V_{A}([\mathbf{u}, \mathbf{v}])=-0.0019<0$. After quite tedious computations one obtains that $A$ is a suitable aggregation function. This example can be generalized for any $n>3$. 
The aggregation function $A$ from the previous example is a 3-quasi-copula. In general, $n$-quasicopulas are functions $Q:[0,1]^{n} \rightarrow[0,1]$, which satisfy the same boundary conditions (C1) as $n$-copulas do and which are non-decreasing (in each variable) and 1-Lipschitz, see [20, 4].

In contrast to $n$-copulas, not all $n$-quasi-copulas are suitable aggregation functions.

Example 2 The function $W:[0,1]^{3} \rightarrow[0,1]$ given by $W(x, y, z)=\max \{0, x+y+z-2\}$ is a proper 3 -quasi-copula. As the $W$-volume

$$
V_{W}([(0.5,0.5,0.5),(1,1,1)])=-0.5<0,
$$

$W$ is not a suitable aggregation function, because Theorem 2 requires the $W$-volume of the 3 -box $[(0.5,0.5,0.5),(1,1,1)]$ to be non-negative.

If we denote by $\mathcal{C}_{(n)}$ the set of all $n$-copulas, by $\mathcal{Q}_{(n)}$ the set of all $n$-quasi-copulas and by $\mathcal{F}_{(n)}$ the set of all $n$-ary aggregation functions suitable for our construction, then, supported by the previous results, we can write $\mathcal{C}_{(n)} \varsubsetneqq \mathcal{F}_{(n)}$ and $\mathcal{Q}_{(n)} \nsubseteq \mathcal{F}_{(n)}$.

\section{Special sets of suitable aggregation functions}

In this section we focus our attention on the set of Archimedean $n$-quasi-copulas. Let us introduce several preparatory notions.

Definition 1 An n-quasi-copula $Q:[0,1]^{n} \rightarrow[0,1]$ given by

$$
Q\left(x_{1}, \ldots, x_{n}\right)=\varphi^{(-1)}\left(\varphi\left(x_{1}\right)+\cdots+\varphi\left(x_{n}\right)\right),
$$

where $\varphi:[0,1] \rightarrow[0, \infty]$ is a continuous strictly decreasing convex function with $\varphi(1)=0$ and pseudoinverse $\varphi^{(-1)}$, is called an Archimedean $n$-quasicopula.

The function $\varphi$ is called an additive generator of $Q$. Its pseudo-inverse $\varphi^{(-1)}:[0, \infty] \rightarrow[0,1]$ is defined by

$$
\varphi^{(-1)}(u)=\varphi^{-1}(\min (\varphi(0), u)) .
$$

For $n$-copulas we have the following result, see [17].

Theorem 3 A function $C:[0,1]^{n} \rightarrow[0,1]$ given by

$$
C\left(x_{1}, \ldots, x_{n}\right)=\varphi^{(-1)}\left(\varphi\left(x_{1}\right)+\cdots+\varphi\left(x_{n}\right)\right),
$$

where $\varphi:[0,1] \rightarrow[0, \infty]$ is a continuous strictly decreasing convex function with $\varphi(1)=0$ and pseudoinverse $\varphi^{(-1)}$, is an n-copula if and only if there exists an $n$-monotone function $f:[-\infty, 0] \rightarrow[0,1]$ such that

$$
\varphi^{(-1)}(-x)=f(x), \quad x \in[-\infty, 0] .
$$

Note that a real function $f$ is called $n$-monotone on an interval $I$ if all its differences of order $1, \ldots, n$ are non-negative. This means that $f$ is $n$-monotone if and only if for each $k \in\{1, \ldots, n\}$, each $x \in I$ and all $\epsilon_{1}, \ldots \epsilon_{k}>0$ such that $x+\epsilon_{1}+\cdots+\epsilon_{k} \in I$

$$
\sum_{I \subseteq\{1, \ldots, k\}} f\left(x+\sum_{i \in I} \epsilon_{i}\right)(-1)^{|I|+k} \geq 0,
$$

compare with $[2,16,11]$. Now, we can formulate the result.

Theorem 4 Let $Q$ be an Archimedean n-quasicopula. Then the following is equivalent

(i) $Q$ is a suitable aggregation function.

(ii) $Q$ is an $n$-copula.

Proof. (ii) $\Rightarrow$ (i). The claim is evident.

(i) $\Rightarrow$ (ii). Let $k \in\{1, \ldots, n\}$. Let $x_{1}, \ldots, x_{k}$ and $x_{1}^{\prime}$ be any elements in $[0,1]$ such that $x_{1}^{\prime}>x_{1}$. Consider the $n$-box $\left[x_{1}, x_{1}^{\prime}\right] \times\left[x_{2}, 1\right] \times \ldots \times\left[x_{k}, 1\right] \times[0,1] \times$ $\ldots \times[0,1]$. By Theorem 2 it holds

$$
V_{Q}\left(\left[x_{1}, x_{2}, \ldots, x_{k}, 0, \ldots, 0\right] \times\left[x_{1}^{\prime}, 1, \ldots, 1\right]\right) \geq 0 .
$$

On the other hand, as 0 is the annihilator of $Q$ and $Q$ is generated by $\varphi(\varphi(1)=0)$, we obtain

$$
\begin{aligned}
& V_{Q}\left(\left[x_{1}, x_{2}, \ldots, x_{k}, 0, \ldots, 0\right] \times\left[x_{1}^{\prime}, 1, \ldots, 1\right]\right) \\
= & \sum_{I \subseteq\{1, \ldots, k\}} \varphi^{(-1)}\left(\sum_{i \in I} \varphi\left(x_{i}\right)+\varphi\left(x_{1}^{\prime}\right) \mathbf{1}_{I^{c}}(1)\right)(-1)^{|I|}
\end{aligned}
$$

If we denote

$$
\begin{gathered}
a=\varphi\left(x_{1}^{\prime}\right), \quad b_{1}=\varphi\left(x_{1}\right)-\varphi\left(x_{1}^{\prime}\right), \\
b_{2}=\varphi\left(x_{2}\right), \ldots, b_{k}=\varphi\left(x_{k}\right),
\end{gathered}
$$

then from (4) and (3) we obtain that for each $k \leq n$ it holds

$$
\sum_{I \subseteq\{1, \ldots, k\}} \varphi^{(-1)}\left(a+\sum_{i \in I} b_{i}\right)(-1)^{|I|} \geq 0 .
$$

For $x \in[-\infty, 0]$ define $f(x)=\varphi^{(-1)}(-x)$ and denote $u=-\left(a+\sum_{i=1}^{k} b_{i}\right)$. Then

$$
\begin{aligned}
\varphi^{(-1)}\left(a+\sum_{i \in I} b_{i}\right) & =f\left(-a-\sum_{i \in I} b_{i}\right) \\
& =f\left(u+\sum_{i \in I} b_{i}\right)(-1)^{k} .
\end{aligned}
$$

Finally, from (5) and (6) it follows that

$$
\sum_{I \subseteq\{1, \ldots, k\}} f\left(u+\sum_{i \in I} b_{i}\right)(-1)^{|I|+k} \geq 0,
$$

which means that $f$ is $n$-monotone and by Theorem $3, Q$ is an $n$-copula. 
Remark 1 Observe that based on the results presented in [25], Theorem 4 can be generalized to the case of associative $n$-quasi-copulas. The associativity of $n$-ary functions in the Post sense [22] is considered, i.e., the associativity of an $n$-ary quasi-copula $Q$ means that for all $x_{1}, \ldots, x_{2 n-1} \in[0,1]$ it holds

$$
\begin{aligned}
& Q\left(Q\left(x_{1}, \ldots, x_{n}\right), x_{n+1}, \ldots, x_{2 n-1}\right) \\
= & Q\left(x_{1}, Q\left(x_{2}, \ldots, x_{n+1}\right), \ldots, x_{2 n-1}\right)=\ldots \\
= & Q\left(x_{1}, \ldots, x_{n-1}, Q\left(x_{n}, \ldots, x_{2 n-1}\right) .\right.
\end{aligned}
$$

An associative $n$-quasi-copula is a suitable aggregation function if and only if it is an ordinal sum of $n$-ary Archimedean copulas. For the later concept see [18].

\section{A universal extending method}

As mentioned in Introduction, the Lovász and Owen extensions can be applied universally, independently of the arity $n$. To obtain another universal extension method, consider a suitable binary aggregation function $A:[0,1]^{2} \rightarrow[0,1]$. In [13] we proved the following characterization of suitable binary aggregation functions. Note that Example 2 shows that this characterization is valid for binary case only.

Theorem 5 Let $A \in \mathcal{A}_{(2)}$. The function $F_{m, A}$ given by (1) is for each $m \in \mathcal{M}_{(2)}$ an aggregation function extending $m$ if and only if for each $(x, y) \in[0,1]^{2}$ it holds

$$
A(x, y)=Q(f(x), g(y))
$$

where $Q$ is a 2-quasi-copula and $f, g$ are nondecreasing $[0,1] \rightarrow[0,1]$ functions with $f(0)=$ $g(0)=0, f(1)=g(1)=1$.

Suppose that the considered suitable binary aggregation function $A$ is associative. The associativity of $A$ means that for all $x, y, z \in[0,1]$ it holds $A(A(x, y), z)=A(x, A(y, z))$, i.e.,

$$
\begin{aligned}
& Q(f(Q(f(x), g(y))), g(z)) \\
= & Q(f(x), g(Q(f(y), g(z)))) .
\end{aligned}
$$

Putting $y=z=1$ one obtains $f(x)=f(f(x))$. Similarly, the equality $g(z)=g(g(z))$ can be proved. To obtain a continuous extension, the continuity of $f$ and $g$ is required, and so the only possibility for $f$ and $g$ is the identity function. Thus $A=Q$, where $Q$ is an associative 2-quasi-copula. Its $n$-ary extension is a suitable aggregation function if and only if it is an $n$-copula. Following the results in [18] we can conclude:

Theorem 6 An associative binary aggregation function $A:[0,1]^{2} \rightarrow[0,1]$ generates a continuous extended aggregation function $\bar{A}: \bigcup_{n \in \mathbb{N}}[0,1]^{n} \rightarrow[0,1]$ such that for each $n \geq 2,\left.\bar{A}\right|_{[0,1]^{n}}$ is a suitable $n$-ary aggregation function, if and only if $A$ is an ordinal sum of Archimedean copulas, $A=\left(\left\langle a_{k}, b_{k}, C_{k}\right\rangle\right)$, where each $C_{k}$ is generated by an additive generator $\varphi_{k}:[0,1] \rightarrow[0, \infty]$, such that the function $f_{k}:[-\infty, 0] \rightarrow[0,1]$, given by $f_{k}(x)=\varphi_{k}^{(-1)}(-x)$, is totally monotone, $i$. e., $f_{k}$ has all derivatives on ]$-\infty, 0[$ which are non-negative.

Example 3 (i) Let $A=T_{0}^{H}$ be the Hamacher product given by $A(x, y)=\frac{x y}{x+y-x y} . \quad A$ is an Archimedean copula generated by the additive (strict) generator $\varphi: \varphi(x)=\frac{1}{x}-1$. The function $f(x)=\varphi^{-1}(-x)=\frac{1}{1-x}, x \in[-\infty, 0]$, has derivatives $f^{(n)}(x)=\frac{n !}{(1-x)^{n+1}}, n \in \mathbb{N}$, which are nonnegative. The function

$$
A\left(x_{1}, \ldots, x_{n}\right)=\left(\sum_{i=1}^{n} \frac{1}{x_{i}}-n+1\right)^{-1}
$$

is a suitable $n$-ary aggregation function and moreover, for any $n$.

(ii) Let $A$ be the copula ordinal sum, $A=$ $(\langle 0,1 / 2, \Pi\rangle)$, i.e.,

$$
A(x, y)= \begin{cases}2 x y & (x, y) \in[0,1 / 2],^{2} \\ \min \{x, y\} & \text { otherwise. }\end{cases}
$$

The product copula $\Pi$ is generated by the additive generator $\varphi(x)=-\log x$. For the function $f(x)=$ $\varphi^{-1}(-x)=e^{x}, x \in[-\infty, 0]$, all derivatives are nonnegative. The function

$$
\begin{aligned}
& A\left(x_{1}, \ldots, x_{n}\right) \\
& =\left\{\begin{array}{cl}
\frac{1}{2} \prod_{i=1}^{n} \min \left\{2 x_{i}, 1\right\} & \text { if } \min \left\{x_{1}, \ldots, x_{n}\right\} \leq \frac{1}{2}, \\
\min \left\{x_{1}, \ldots, x_{n}\right\} & \text { otherwise, }
\end{array}\right.
\end{aligned}
$$

is a suitable aggregation function for any $n$.

Observe that the extension of fuzzy measures based on $A$ can be seen as a mixture of the Lovász and Owen extensions in the following sense: if $\mathbf{x} \in$ $[1 / 2,1]^{n}$ then $F_{m, A}(\mathbf{x})=F_{m, M i n}(\mathbf{x})$, i.e., $F_{m, A}$ is just the Lovász extension, and if $\mathbf{x} \in[0,1 / 2]^{n}$ then $F_{m, A}(\mathbf{x})=\frac{1}{2} F_{m, \Pi}(2 \mathbf{x})$, i.e., $F_{m, A}$ is a linear transform of the Owen extension.

Acknowledgment The authors acknowledge the support of the grant VEGA 1/0198/09 and the project of Science and Technology Assistance Agency under the contract No. APVV-0012-07.

\section{References}

[1] C. Alsina, R.B. Nelsen, B. Schweizer, On the characterization of a class of binary operations on distributions functions, Stat. Probab. Lett., 17:85-89, 1993.

[2] A. G. Bronevich: On the closure of families of fuzzy measures under eventwise aggregation, Fuzzy Sets and Systems, 153:45-70, 2005. 
[3] T. Calvo, A. Kolesárová, M. Komorníková, R. Mesiar, Aggregation Operators: Properties, Classes and Construction Methods. In Aggregation Operators. New Trends and Applications, T. Calvo, G. Mayor, R. Mesiar, eds. PhysicaVerlag, Heidelberg, pp. 3-107, 2002.

[4] I. Cuculescu, R. Theodorescu, Copulas: diagonals and tracks, Revue Rumaine de Mathématique Pures et Appliquées, 46:731-742, 2001.

[5] A. Chateauneuf, J.Y. Jaffray, Some characterizations of lower probabilities and other monotone capacities through the use of Möbius inversion, Math. Soc. Sciences, 17:263-283, 1989.

[6] G. Choquet, Theory of capacities, Ann. Inst. Fourier, 5:131-295, 1953-54.

[7] C. Genest, J.J. Quesada Molina, J.A. Rodríguez-Lallena, and C. Sempi, A characterization of quasi-copulas, J. Multivariate Anal., 69:193-205, 1999.

[8] M. Grabisch, T. Murofushi, and M. Sugeno, Fuzzy Measures and Integrals. Theory and Applications, Physica Verlag, Heidelberg, 2000.

[9] M. Grabisch, J.-L. Marichal, R. Mesiar, and E. Pap, Aggregation Functions, Cambridge University Press, Cambridge, 2009.

[10] E.P. Klement, R. Mesiar, and E. Pap, Triangular norms, Kluwer Academic Publishers, Dordrecht, 2000.

[11] E.P. Klement, M. Manzi, R. Mesiar, Aggregation functions with stronger types of monotonicity. In E. Hüllermeier, R. Kruse, F. Hoffmann, eds., Proc. IPMU 2010, LNAI 6178, Springer-Verlag, Berlin Heidelberg, pp. 418424, 2010.

[12] A. Kolesárová, 1-Lipschitz aggregation operators and quasi-copulas, Kybernetika , 9:615629, 2003.

[13] A. Kolesárová, A. Stupňanová, and J. Beganová, Aggregation-based extensions of utility functions. Fuzzy Sets and Systems, submitted.

[14] L. Lovász, Submodular function and convexity. In Mathematical Programming: The state of the art. Springer, Berlin, pp. 235-257, 1983.

[15] J.-L. Marichal, Aggregation of interacting criteria by means of the discrete Choquet integral. In Aggregation Operators. New Trends and Applications, T. Calvo, G. Mayor, R. Mesiar, eds. Physica-Verlag, Heidelberg, pp. 224-244, 2002.

[16] M. Marinacci, L. Montrucchio: Ultramodular functions, Math. Oper. Res., 30:311-332, 2005.

[17] A.J. McNeil, J. Nešlehová, Multivariate Archimedean copulas, d-monotone functions and $l_{1}$-norm symmetric distributions, The $A n$ nals of Statistics, 37:3059-3097, 2009.

[18] R. Mesiar, C. Sempi, Ordinal sums and idempotents of copulas, Aequationes Mathematicae, 79:39-52 2010.

[19] R.B. Nelsen, An Introduction to Copulas, Lecture Notes in Statistics 139, Springer Verlag,
New York, 1999.

[20] R.B. Nelsen, J.J. Quesada-Molina, B. Schweizer, and C. Sempi, Derivability of some operations on distributions functions. In L. Rüschendorf, B. Schweizer, M.D. Taylor, eds., Distributions with Fixed Marginals and Related Topics, CA: IMS Lecture Notes Monograph Series Number 28, Hayward, pp. 233-243, 1996.

[21] G. Owen, Multilinear extensions of games. In A.E. Roth, ed., The Shapley value. Essays in Honour of Lloyd S. Shapley, Cambridge University Press, pp. 139-151, 1988.

[22] E.-L. Post, Polyadic groups, Trans. Amer. Math. Soc., 48:208-350, 1940.

[23] D. Schmeidler, Integral representation without additivity, Proc. Amer. Math. Soc., 97:255-261, 1986.

[24] A. Sklar, Fonctions de répartition à $n$ dimensions et leurs marges, Publ. Inst. Statist. Univ. Paris, 8:229-231, 1959.

[25] A. Stupňanová, A. Kolesárová, Associative $n$-dimensional copulas. Kybernetika, in press, 2011.

[26] Z. Wang, G.J. Klir, Fuzzy Measure Theory, Plenum Press, New York, 1992. 